\title{
Detection of Sensor Faults in Autonomous Helicopters*
}

\author{
Guillermo Heredia, Aníbal Ollero, Rajesh Mahtani and \\ Manuel Béjar \\ Robotics, Computer Vision and Intelligent Control Group \\ Dept. Ingeniería de Sistemas y Automática, University of Seville \\ Camino de los Descubrimientos s/n, 41092, Seville, Spain \\ \{guiller,aollero,rajesh,mbejar\}@cartuja.us.es \\ http://www.esi.us.es/GVR
}

\begin{abstract}
This paper presents a sensor fault detection and diagnosis system for autonomous helicopters. The system has been tested with the MARVIN autonomous helicopter. Fault detection is accomplished by evaluating any significant change in the behaviour of the vehicle with respect to the fault-free behaviour, which is estimated by using an observer. The effectiveness of the proposed approach is demonstrated by means of MARVIN experimental results.
\end{abstract}

Index Terms - Autonomous helicopters; UAV; fault detection; fault diagnosis; sensors.

\section{INTRODUCTION}

Unmanned Aerial Vehicles (UAV) are increasingly used in many applications in which ground vehicles cannot access to the desired locations due to the characteristics of the terrain and the presence of obstacles. In many cases the use of aerial vehicles is the best way to approach the objective to get information or to deploy instrumentation.

The work described in this paper has been performed in the framework of the COMETS project funded by the IST Programme of the European Union, devoted to the coordination and control of multiple unmanned aerial vehicles. Particularly, both helicopters and airship have been integrated in the COMETS system.

Helicopters have high maneuverability and hovering ability. Then, they are well suited to agile target tracking tasks, as well as to inspection and monitoring tasks that require to maintain a position and to obtain detailed views. Furthermore, the vertical take-off and landing of helicopters is very desirable in many applications. Remotely piloted helicopters are inherently unstable and dynamically fast. Even with improved stability augmentation devices, a skilled, experienced pilot is required to control them during flight. Autonomous helicopter control is a challenging task involving a multivariable nonlinear open-loop unstable system with actuator saturations.

Moreover, helicopters do not have the graceful degradation properties of fixed wing aircrafts or airships in case of failures. Thus, a failure in any part of the autonomous helicopter (sensors, actuators, control system, etc) can be catastrophic. If the failure is not detected and accounted for, the helicopter may crash.

Fault Detection and Isolation (FDI) techniques have been widely used in process industry to detect faults in sensors and actuators. If a fault is detected, the structure of

\author{
Volker Remuß and Marek Musial \\ Institut für Technische Informatik und Mikroelektronik(TIME) \\ Technical University of Berlin \\ Einsteinufer 17, D-10587 Berlin, Germany
}

\{remuss,musial\}@cs.tu-berlin.de 
This helicopter is described in section II. Section III presents the fault detection and isolation approach. The results on the application of these techniques to MARVIN are presented in section IV. Sections V and VI are devoted to the conclusions and references.

\section{THE MARVIN HELICOPTER}

The basis of MARVIN (Fig. 1) is a conventional model helicopter. It has a rotor diameter of $1.9 \mathrm{~m}$ and is equipped with a two-stroke petrol engine producing about 2 $\mathrm{kW}$. Its takeoff weight amounts to about $12 \mathrm{~kg}$, operation time is approximately 30 minutes. Figure 1 shows a view of MARVIN in flight. The sensors on board of MARVIN are:

1. A custom-built inertial measurement unit consisting of three magnetometers, three semiconductor accelerometers, and three piezo-electric gyroscopes.

2. A NovAtel RT-2 carrier phase differential GPS receiver.

3. A fire sensor that detects a certain range of ultraviolet light characteristic of burning wood, gas, or oil.

4. Temperature sensor to measure the heat of surrounding air.

5. A light barrier rpm sensor for measuring main rotor rpm.

6. An ultrasonic rangefinder looking down.

7. A high resolution digital still camera.

On-board data processing is divided between a PCarchitecture single-board computer (by Compulab) and an Infineon SAB80C167 microcontroller. The $\mathrm{PC}$ is responsible for wireless network communication and for the interfacing of the on-board camera, while the microcontroller is used for sensor and actuator data processing and the implementation of the flight controller.

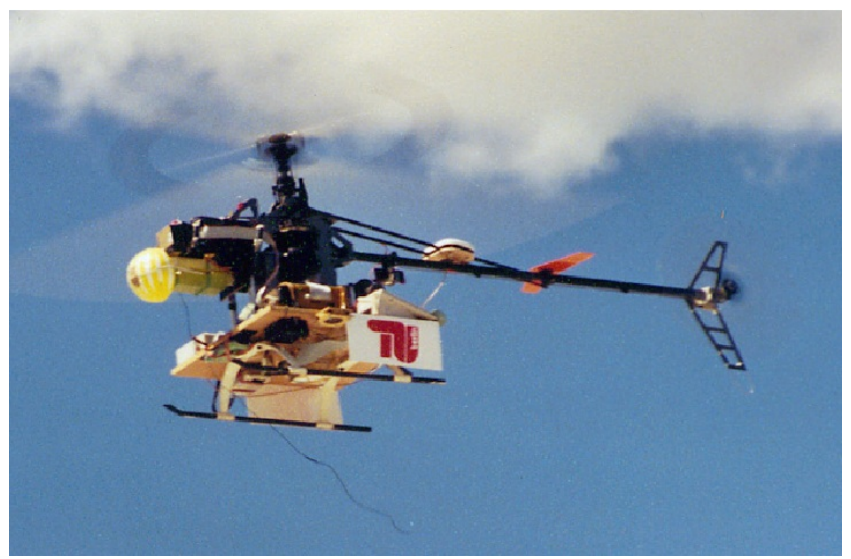

Fig. 1. MARVIN in flight.

\section{SENSOR FAULT DETECTION AND ISOLATION}

\section{A. Sensor fault detection structure.}

The helicopter sensor FDI subsystem performs the tasks of failure detection and identification by continuously monitoring the outputs of the sensors. Under nominal conditions, these measurements follow predictable patterns, within a tolerance determined by the amount of uncertainties introduced by random system disturbances and measurement noise in the sensors. Usually, sensor FDI tasks are accomplished by observing when the output of a failed sensor deviates from its predicted pattern.

For detection of faults in the helicopter sensors, a bank of output estimators has been implemented as showed in Fig. 2. The number of these estimators is equal to the number of system outputs. Thus, each device is driven by a single output and all the inputs of the system. In this case a fault on the $i$ th output sensor affects only the residual function of the output observer or filter driven by the $i$ th output.

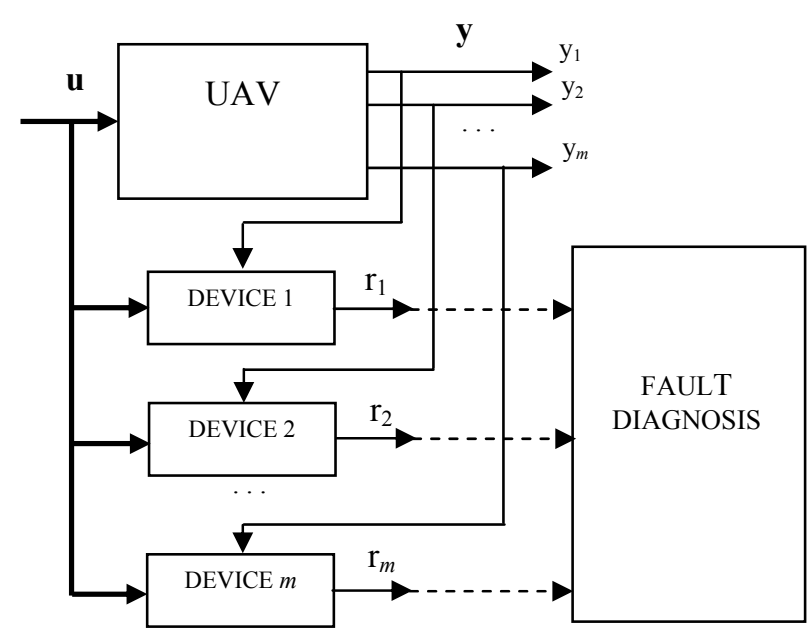

Fig. 2. Bank of estimators for output residual generation.

A residual is generated for each sensor, comparing the estimator output with the sensor output. Each residual is not affected by the other sensors, and therefore fault identification is straightforward: each residual is only sensitive to a single helicopter sensor. If the residual $k$ goes above the threshold level, a fault has been detected in sensor $k$.

The FDI system with the above structure has been implemented using ARX input-output models. The results are presented in the following subsections.

\section{B. Sensor failure types.}

Sensors used in autonomous helicopters can fail in several ways. Some failure types are general for various sensors, while others are specific of a single sensor. The failure types that have been considered are:

1) Total sensor failure. This is a catastrophic failure, at a given time the sensor stops working and gives a constant zero output after that. This failure can be due to electrical or communication problems.

2) Stuck with constant bias sensor failure. In this failure type, at a given time the sensor gets stuck with a constant bias, and the output remains constant.

3) Drift or additive-type sensor failure. This is a very common failure in analog sensors. Due to internal temperature changes or calibration problems, the sensor output has an added constant term (the drift).

4) Multiplicative-type sensor failure. This failure type is caused by a scaling error in the sensor output (a multiplicative factor is applied to the sensor nominal value).

5) Outlier data sensor failure. This is a failure that appears sometimes in GPS sensors. It is a temporal failure: a 
single point with a large error is given by the GPS sensor, but after that, the following measurements are correct. This error can be caused by failures in the GPS internal signal processing algorithms, temporary satellite signal blocking, ...

\section{B. Observer design.}

The estimation of the state vector is unnecessary for FDI purposes and only the output estimation is required [8]. A residual generator based on a Luenberger observer can be used.

As only the outputs are required for FDI, an inputoutput model of the helicopter system can be identified for output prediction. Since past inputs and outputs are available to the FDI system at any given instant, the inputoutput model can be used to estimate the actual output in fault-free conditions. A linear mathematical model of the input-output links can be used for local analysis. This model can be obtained by means of well known identification schemes. When the signal to noise ratios are high Auto Regressive eXogenous (ARX) models can be used. The ARX model is chosen with the structure that achieve the smallest Akaike's Information Theoretic Criterion (AIC) [12], according to a simple search algorithm, in which the first half of data is used for estimation and the second for cross validation.

\section{Residual generation.}

Independent residuals are constructed for each different sensor failure. Residuals are designed so that they respond to an individual failure and not to the others. In general, residuals $R_{k}$ are functions of the squared difference between real $\left(c_{i}\right)$ and estimated $\left(\hat{c}_{i}\right)$ sensor outputs:

$$
R_{k}=\sum_{i=1}^{n} m_{i}\left(c_{i}-\hat{c}_{i}\right)^{2}
$$

where $m_{i}$ are weighting coefficients that are determined for each failure based on experience and experimentation.

The first time the residual goes above the threshold level, the fault is supposed to be present.

\section{FAULT DETECTION RESULTS}

In this section, the results of MARVIN sensor FDI system using linear ARX output estimators are presented. All the experiments presented in this section have been done using real MARVIN flight data.

Flight data were recorded from several experiments carried out at the Lousá (Portugal) airfield during the general experiments of the COMETS project. These experiments were performed in spring with temperatures around $20^{\circ} \mathrm{C}$ and low wind conditions.

The MARVIN helicopter has 12 individual sensors: 3 gyroscopes, 3 accelerometers, 3 components of the magnetic sensor and the 3 GPS coordinates. Since there are too many possible combinations of sensors and failure types, only a few representative cases will be described in detail in this section.

The $\mathrm{z}$ component of the gyroscope angular velocity sensor will be used as representative case of sensor failure detection for the first four failure types presented (total sensor failure, stuck with constant bias, additive type and multiplicative type). The $\mathrm{z}$ component of the GPS sensor will be used for the fifth failure type (outlier data), since it is a specific failure of GPS sensors.

For some failure types, a sensor failure detectability study for all twelve MARVIN sensors has been conducted. Sensor failures have been studied in nine different helicopter experiments, using real MARVIN flight data. The results have been summarized in tables, including the detection time.

\section{A. Total sensor failure detection.}

A total sensor fault has been reproduced using MARVIN flight data. In the upper plot of Fig. 3 the nominal sensor data are shown with dashed lines, while the sensor output is represented with solid line. It can be seen that at $\mathrm{t}=18 \mathrm{~s}$. the sensor output goes to zero, and remains null for the rest of the experiment.

The gyro-z residual signal is shown in the lower plot. It can be seen that shortly after the fault (one sampling period), the residual goes above the threshold level (dashed line). The fault is detected almost immediately.

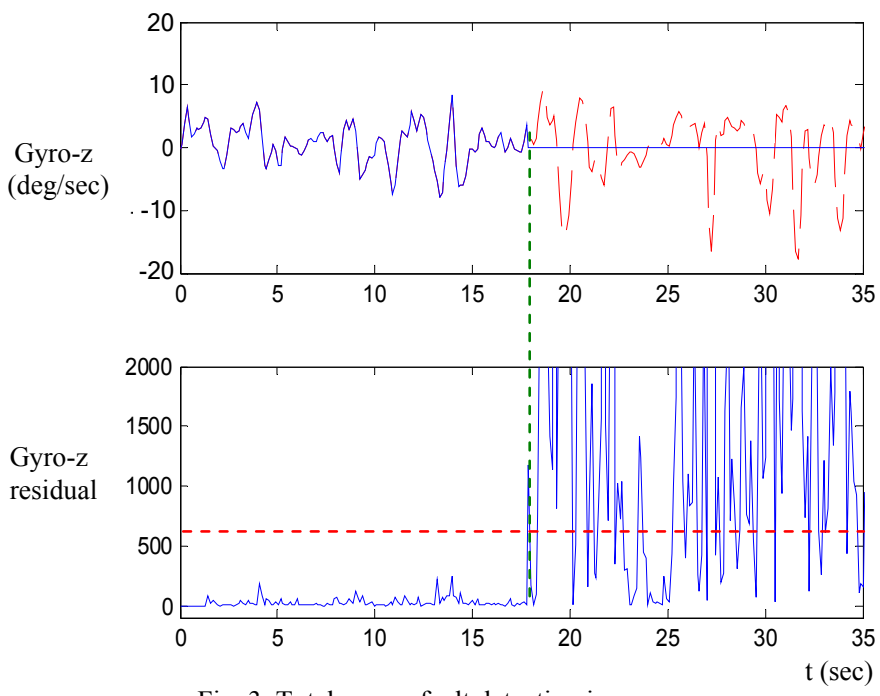

Fig. 3. Total sensor fault detection in gyro-z.

\section{B. Stuck with constant bias sensor failure detection.}

In this case, the gyro-z sensor output gets stuck with the last output value before the fault produced. In Fig. 4 a fault has been reproduced in the gyro-z sensor at $\mathrm{t}=18 \mathrm{~s}$ (upper plot). It can be seen that, shortly after the fault, the residual goes above the threshold level and the fault is detected (lower plot).

For the stuck with constant bias failure type, a detectability study has been done. For each sensor (3 components of gyroscopes, accelerometers, magnetic sensor and GPS), a sensor fault has been produced. In most sensor faults, the faults have been successfully detected in all experiments.

There is an exception: in two experiments, the GPS fault detection system gave false alarms (faults were detected when no fault was present). This is due to GPS output degradation in these two experiments because of satellite signal blocking. MARVIN GPS sensor has an "output quality signal", which gives a measure of GPS 
output error. This signal can be used to discard these false alarms, because the detection thresholds have been set assuming good quality GPS output. The GPS output in these two experiments will have the same problems with the other failure types.

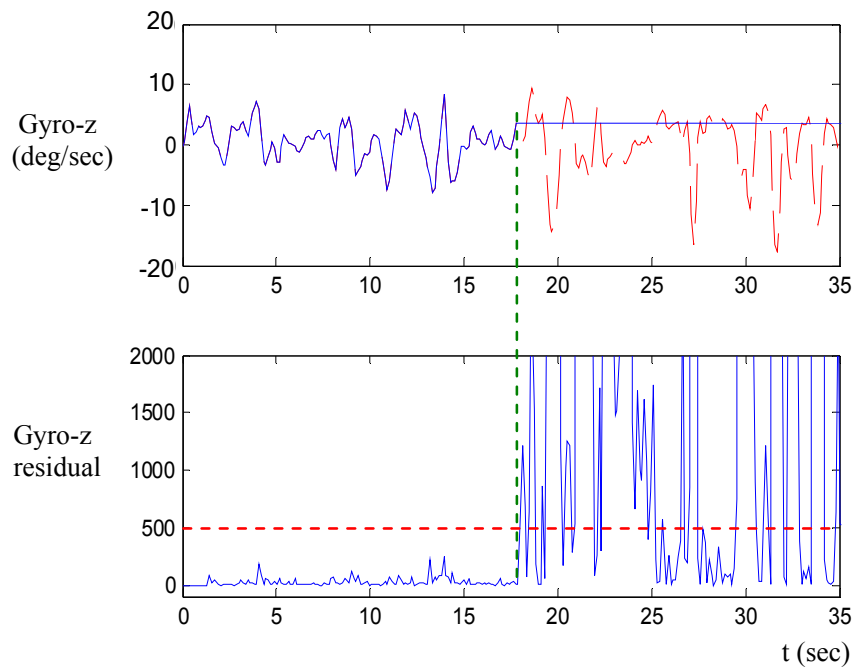

Fig. 4. Stuck with constant bias gyro-z failure detection.

\section{Additive-type sensor failure detection.}

Fig. 5 and Fig. 6 show the results of the fault detection of additive faults in gyro-z sensor output. In Fig. 5 a 3.5 degrees/sec drift has been added, and the residual detects the fault very fast. In Fig. 6 a 1.75 degrees/sec drift has been added to the sensor output at $\mathrm{t}=18 \mathrm{sec}$. In this case, the detection time is larger.

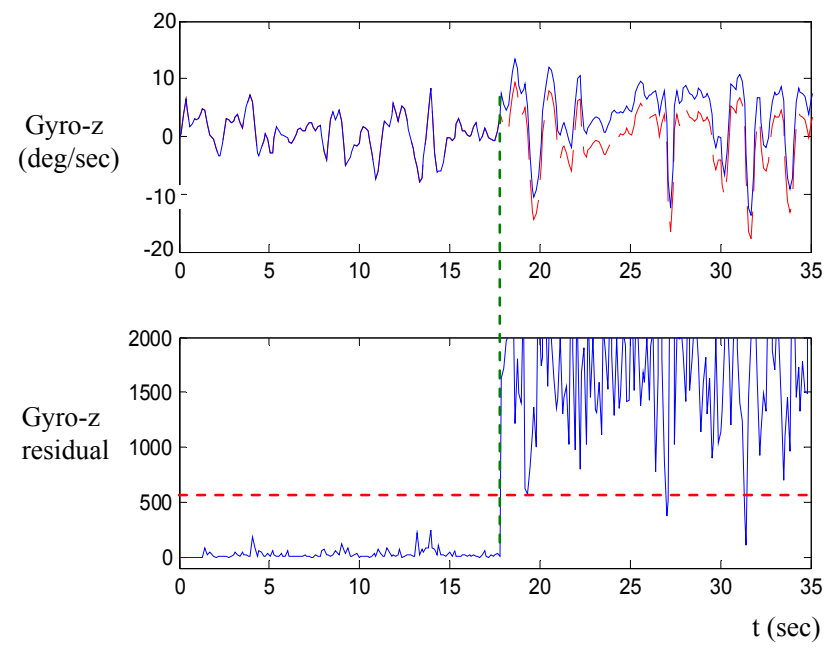

Fig. 5. Additive gyro- $z$ sensor failure detection (drift of +3.5 degrees $/ \mathrm{sec}$ ).
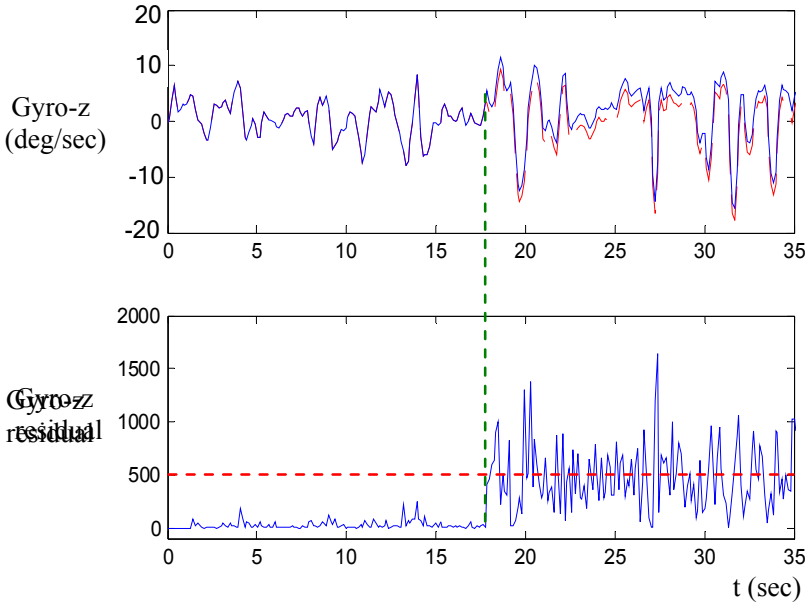

Fig. 6. Additive gyro-z sensor failure detection (drift of +1.75 degrees $/ \mathrm{sec}$ ).

A detectability study has also been done with the additive type sensor failure. Two cases have been considered: a drift of $50 \%$ of the maximum sensor value, which results are presented in Table I, and a drift of $10 \%$ of the maximum sensor value, which results are presented in Table II.

TABLE I

SENSOR FAUlt DETECTION WiTH DRIFT OF 50\% OF MAXIMUM SENSOR VALUE.

\begin{tabular}{|l|c|c|c|c|c|c|c|c|c|c|c|c|}
\hline & \multicolumn{3}{|c|}{ GPS } & \multicolumn{3}{c|}{ ACCEL } & \multicolumn{3}{c|}{ MAG } & \multicolumn{3}{c|}{ GYRO } \\
\hline & $\mathrm{X}$ & $\mathrm{Y}$ & $\mathrm{Z}$ & $\mathrm{X}$ & $\mathrm{Y}$ & $\mathrm{Z}$ & $\mathrm{X}$ & $\mathrm{Y}$ & $\mathrm{Z}$ & $\mathrm{X}$ & $\mathrm{Y}$ & $\mathrm{Z}$ \\
\hline Mean detection time & 0.00 & 0.00 & 0.00 & 0.00 & 0.00 & 0.09 & 0.09 & 0.00 & 0.00 & 0.12 & 0.00 & 0.00 \\
\hline Undetected faults & 0 & 0 & 0 & 0 & 0 & 0 & 0 & 0 & 0 & 0 & 0 & 0 \\
\hline False alarms & 2 & 2 & 0 & 0 & 0 & 0 & 0 & 0 & 0 & 0 & 0 & 0 \\
\hline
\end{tabular}

The results presented in Table I show that a drift 50\% of maximum sensor value can be detected reliably in almost all the experiments (except with GPS in the two experiments mentioned in the above subsection). It can be noticed that the detection time is very short. The detection time means the time between the first erroneous measurement and the failure detection, excluding the processing time.

On the other hand, the results of Table II show that a $10 \%$ drift is much harder to detect. There is a number of undetected faults and false alarms, making the detection of this kind of faults unreliable. Detection times are also much larger.

TABLE II.

SENSOR FAULT Detection With DRIFT OF 10\% OF MAXIMUM SENSOR VALUE

\begin{tabular}{|l|c|c|c|c|c|c|c|c|c|c|c|c|}
\hline & \multicolumn{3}{|c|}{ GPS } & \multicolumn{2}{c|}{ ACCEL } & \multicolumn{3}{c|}{ MAG } & \multicolumn{3}{c|}{ GYRO } \\
\hline & $\mathrm{X}$ & $\mathrm{Y}$ & $\mathrm{Z}$ & $\mathrm{X}$ & $\mathrm{Y}$ & $\mathrm{Z}$ & $\mathrm{X}$ & $\mathrm{Y}$ & $\mathrm{Z}$ & $\mathrm{X}$ & $\mathrm{Y}$ & $\mathrm{Z}$ \\
\hline $\begin{array}{l}\text { Mean detection } \\
\text { time }\end{array}$ & 0.15 & 3.67 & 0.00 & 0.75 & 2.42 & 0.75 & 0.14 & 0.00 & 0.00 & 0.75 & 3.25 & 0.90 \\
\hline $\begin{array}{l}\text { Undetected } \\
\text { faults }\end{array}$ & 0 & 0 & 0 & 0 & 0 & 1 & 0 & 0 & 0 & 2 & 2 & 1 \\
\hline False alarms & 2 & 2 & 2 & 2 & 0 & 3 & 3 & 0 & 0 & 0 & 1 & 0 \\
\hline
\end{tabular}




\section{Multiplicative-type sensor failure detection.}

Fig. 7 shows the results of the fault detection of multiplicative fault in gyro-z sensor output. When the fault happens, the sensor output is only $50 \%$ of the nominal output. In the experiment shown, the detection time is not very good, but this is due to the fact that just after the fault is declared, the gyro sensor outputs are close to zero, and it is difficult to distinguish the fault from measurement noise. When the angular velocities get larger, the fault is easily detected.

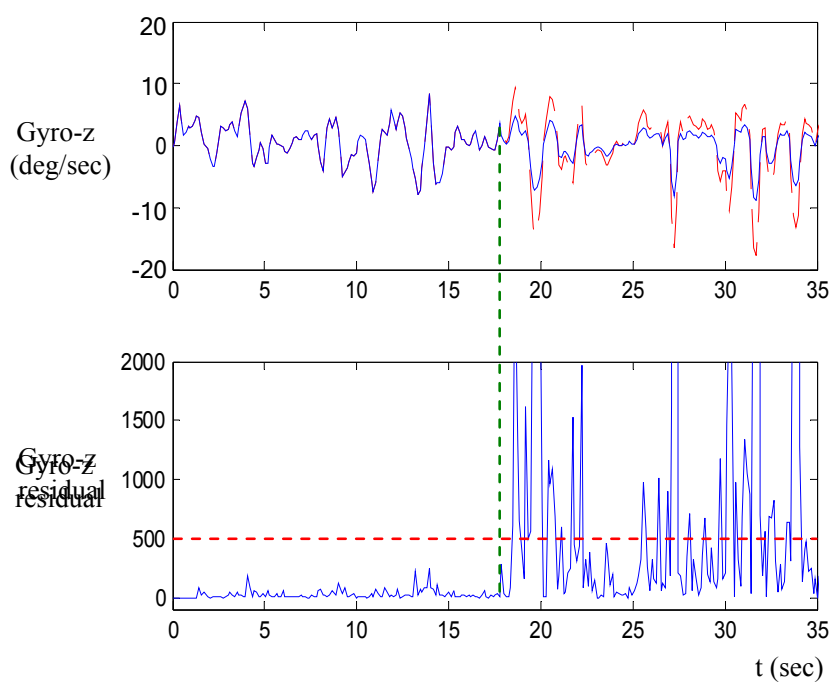

Fig. 7. Multiplicative gyro-z sensor failure detection $(50 \%$ of nominal output).

A detectability study has also been done with the multiplicative type sensor failure. Two cases have been considered: a sensor output of $50 \%$ of the nominal output, which results are presented in Table III, and a sensor output of $10 \%$ of the nominal output, which results are presented in Table IV.

TABLE III

SENSOR FAULT DETECTION WITH A SENSOR OUTPUT OF 50\% OF THE NOMINAL OUTPUT.

\begin{tabular}{|l|c|c|c|c|c|c|c|c|c|c|c|c|}
\hline & \multicolumn{3}{|c|}{ GPS } & \multicolumn{3}{c|}{ ACCEL } & \multicolumn{3}{c|}{ MAG } & \multicolumn{3}{c|}{ GYRO } \\
\hline & $\mathrm{X}$ & $\mathrm{Y}$ & $\mathrm{Z}$ & $\mathrm{X}$ & $\mathrm{Y}$ & $\mathrm{Z}$ & $\mathrm{X}$ & $\mathrm{Y}$ & $\mathrm{Z}$ & $\mathrm{X}$ & $\mathrm{Y}$ & $\mathrm{Z}$ \\
\hline $\begin{array}{l}\text { Mean detection } \\
\text { time }\end{array}$ & 0.98 & 0.00 & 2.29 & 1.39 & 0.40 & 4.52 & 1.12 & 0.00 & 0.00 & 1.47 & 3.27 & 4.11 \\
\hline $\begin{array}{l}\text { Undetected } \\
\text { faults }\end{array}$ & 0 & 0 & 0 & 0 & 0 & 0 & 0 & 0 & 0 & 0 & 0 & 0 \\
\hline False alarms & 2 & 2 & 1 & 0 & 0 & 0 & 0 & 0 & 0 & 0 & 0 & 0 \\
\hline
\end{tabular}

The results presented in Table III show that a $50 \%$ multiplicative fault can be detected reliably in almost all the experiments (except with GPS in the two experiments mentioned in the above subsection). The detection time is larger than in the additive type sensor failure.

The results of Table IV show that a $10 \%$ multiplicative fault can also be detected with high reliability. The detection times are remarkably close to Table III, indicating that multiplicative faults are detected whenever the sensor output get high positive or negative values, therefore the multiplicative error can be distinguished from sensor noise.
TABLE IV

SENSOR FAULT DETECTION WITH SENSOR OUTPUT OF $10 \%$ OF THE NOMINAL OUTPUT.

\begin{tabular}{|l|c|c|c|c|c|c|c|c|c|c|c|c|}
\hline & \multicolumn{3}{|c|}{ GPS } & \multicolumn{3}{c|}{ ACCEL } & \multicolumn{3}{c|}{ MAG } & \multicolumn{3}{c|}{ GYRO } \\
\hline & $\mathrm{X}$ & $\mathrm{Y}$ & $\mathrm{Z}$ & $\mathrm{X}$ & $\mathrm{Y}$ & $\mathrm{Z}$ & $\mathrm{X}$ & $\mathrm{Y}$ & $\mathrm{Z}$ & $\mathrm{X}$ & $\mathrm{Y}$ & $\mathrm{Z}$ \\
\hline $\begin{array}{l}\text { Mean detection } \\
\text { time }\end{array}$ & 0.98 & 1.25 & 5.81 & 0.88 & 0.27 & 4.51 & 1.12 & 0.00 & 0.00 & 2.81 & 3.25 & 4.16 \\
\hline $\begin{array}{l}\text { Undetected } \\
\text { faults }\end{array}$ & 0 & 0 & 0 & 0 & 0 & 0 & 0 & 0 & 0 & 0 & 0 & 0 \\
\hline False alarms & 2 & 2 & 0 & 0 & 0 & 0 & 0 & 0 & 0 & 0 & 0 & 0 \\
\hline
\end{tabular}

\section{E. Outlier data sensor failure detection.}

This is a failure that appears in GPS receiver data. It is a single data point with large error. Fig. 8 shows the GPS sensor $\mathrm{z}$ data. At $\mathrm{t}=18 \mathrm{sec}$, the output has a $1 \mathrm{~m}$. error added. The fault is easily detected, due to the quality of MARVIN GPS sensor data.

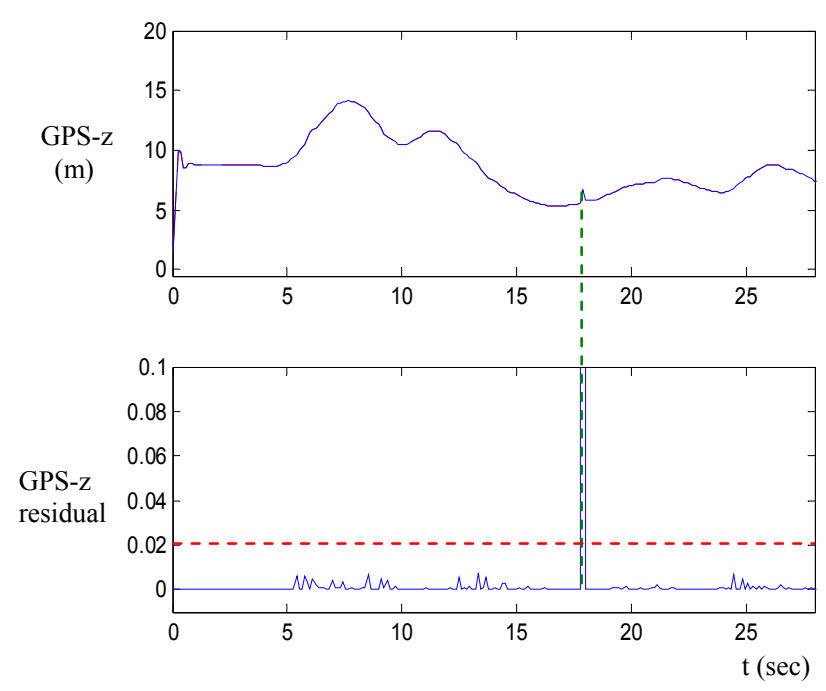

Fig. 8. Outlier data GPS-z sensor failure detection using ARX output estimator $(1 \mathrm{~m}$.)

\section{CONCLUSIONS}

The use of autonomous helicopters in civilian applications requires the improvement of safety conditions to avoid potential accidents. Fault Detection and Isolation plays an important role in this context. This paper has presented a system for helicopter's sensors fault detection and its application to the MARVIN autonomous helicopter. Experiments with MARVIN have been conducted to collect input-output data in many different flight conditions. Five different failure types have been considered. "Hard" failures (zero or constant sensor output) are easily detected by the fault detection system in short time. "Soft" failures (sensor output with additive or multiplicative error) are detected depending on the error size. If errors are too small, they can not be distinguished from noise. Outlier data sensor failures, typical of GPS receivers, are also easily detected. This can 
be combined with GPS signal quality measures that are reported by many GPS receivers to improve position estimation reliability.

\section{ACKNOWLEDGMENTS}

The authors acknowledge the cooperation of the other participants in the COMETS and CROMAT projects.

\section{REFERENCES}

[1] Napolitano M., Y. An, B. Seanor, S. Pispistos and D. Martinelli (1999). Application of a neural sensor validation scheme to actual Boeing B737 flight data. Proc. Of the '99 AIAA Guidance, Navigation and Control Conference.

[2] Napolitano M., Y. An and B. Seanor (2000). A fault tolerant flight control system for sensor and actuator failures using neural networks. Aircraft Design, 3, 103-128.

[3] Napolitano M., D. Windon, J. Casanova, M. Innocenti and G. Silvestri (1998). Kalman filters and neural-network schemes for sensor validation in flight control systems. IEEE Trans. On Control Systems Technology, 6, 596-611.
[4] Rago, C., R. Prasanth, R.K. Mehra and R. Fortenbaugh. (1998). Failure detection and identification and fault tolerant control using the IMMKF with applications to the Eagle-Eye UAV. Proc. Of the $37^{\text {th }}$ Conf. on Decision and Control, Tampa, Florida, USA.

[5] Alessandri, A., M. Caccia and G. Veruggio (1999). Fault detection of actuator faults in unmanned underwater vehicles. Control Engineering Practice, 7, pp. 357-368.

[6] Heredia, G., V. Remuß, A. Ollero, R. Mahtani and M. Musial (2004). Actuator fault detection in autonomous helicopters. Proc. of the $5^{\text {th }}$ IFAC/EURON Symposium on Intelligent Autonomus Vehicles (IAV2004), Lisbon (Portugal).

[7] Gertler, J. (1997). Fault detection and isolation using parity relations. Control Engineering Practice, 5, pp. 653-661.

[8] Patton, R.J. and J. Chen. (1997). Observer-based fault detection and isolation: robustness and applications. Control Engineering Practice, 5, pp. 671-682.

[9] Isermann, R. (1984). Process fault detection based on modelling and estimation methods-a survey. Automatica, 20(4), 387-404.

[10]Patton, R.J., F J Uppal and C J Lopez-Toribio, (2000). Soft Computing Approaches to Fault Diagnosis For Dynamic Systems: A Survey. 4th IFAC Symposium on Fault Detection Supervision and Safety for Technical Processes, Budapest, 14-16 June 2000, 1, pp 298-311.

[11]Isermann, R. and P. Ballé (1997). Trends in the application of modelbased fault detection and diagnosis of technical processes. Control Engineering Practice, 5, pp. 709-719.

[12]Ljung, L. (1999). System Identification - Theory for the User. PrenticeHall, N.J., 2nd edition. 